\title{
Perforation of intrathoracic colon causing acute pneumothorax
}

\author{
BA PRICE, MJ ELLIOTT, G FEATHERSTONE, A BLESOVSKY \\ From the Regional Cardiothoracic Centre, Freeman Hospital, Newcastle upon Tyne
}

Traumatic diaphragmatic hernias, although not uncommon, present infrequently enough to cause diagnostic difficulty, particularly in the acute phase. Blunt injuries account for more diaphragmatic ruptures than direct trauma and the ruptures are usually left sided. The case presented here is reported because of its dramatic manifestation, several years after the initial injury.

\section{Case report}

A 41-year old steel rigger first presented to another hospital on 25 June 1975, having been attacked by a man with a knife. He had sustained five lacerations including three on the chest. One was $6 \mathrm{~cm}$ long, low down on the left posterior wall of the thorax; one was $1.25 \mathrm{~cm}$ long, just below the right nipple; and another was $1.5 \mathrm{~cm}$ long, just anterior to the left anterior axillary line. The other lacerations were over the extensor aspect of the right elbow and over the left palmar thenar eminence. All the lacerations were diagnosed as being superficial with no evidence of intrathoracic or intra-abdominal injury. The wounds were therefore cleaned and sutured and $0.5 \mathrm{ml}$ of tetanus toxoid was administered subcutaneously. The patient was discharged at his own request after observation overnight. All lacerations healed uneventfully and a chest radiograph taken at follow up on 12 August 1975 was normal.

One week before admission to our unit he was admitted to a different hospital complaining of acute left hypochondrial pain. A diagnosis of a large left sided diaphragmatic hernia was made, but before treatment could be instituted the patient again discharged himself from hospital.

On 10 August 1982 he was admitted to the regional cardiothoracic centre with a history of increasing severity of the left hypochondrial pain and the onset of dyspnoea over the preceding $\mathbf{4 8}$ hours. Clinically he was found to be cyanosed and orthopnoeic with pronounced expiratory wheeze. The pulse rate was 110 beats/min with sinus rhythm and a small pulse volume; his arterial blood pressure was $160 / 80 \mathrm{~mm} \mathrm{Hg}$. There was appreciable tracheal shift to the right and there were no breath sounds over the left chest. A chest radiograph (fig 1) confirmed the diagnosis of left tension pneumothorax and showed free fluid in

Address for reprint requests: Mr MJ Elliott, Regional Cardiothoracic Centre, Freeman Hospital, Newcastle. upon Tyne NE7 7DN.

Accepted 10 July 1983

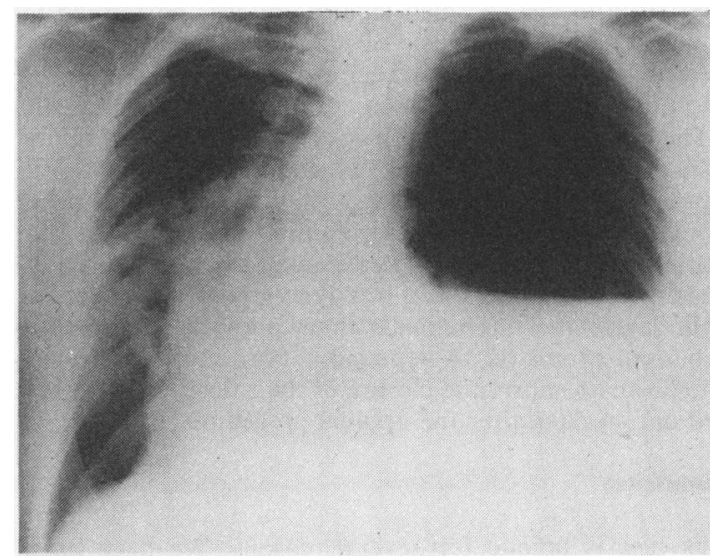

Fig 1 Chest radiograph on admission showing left sided tension pneumothorax.

the pleura: An intercostal drain was inserted into the left upper chest anteriorly and copious quantities of foul smelling flatus were drained under pressure, followed by faecal fiuid. This led to immediate improvement in the clinical state and the radiographic findings (fig 2).

Left thoracotomy was then performed through the bed of the sixth rib. Pus and faeces were removed from the

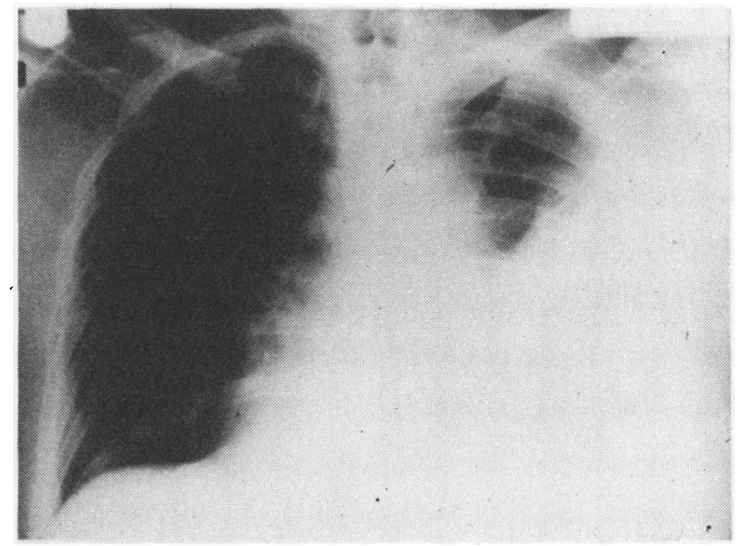

Fig 2 Chest radiograph after insertion of a left intercostal drain. 
pleural cavity. A $12 \mathrm{~cm}$ length of gangrenous colon and an accompanying mass of oedematous omentum were observed to have been trapped through a $4 \mathrm{~cm}$ defect in the posterolateral diaphragm opposite a defect in the eighth intercostal space posteriorly, to which a plug of omentum was adherent. The lung was displaced anterosuperiorly and was covered by a thick fibrinous exudate. Thirty centimetres of colon (including the gangrenous segment) with attached omentum was resected with end to end anastomosis in two layers. The defect in the diaphragm was repaired with interrupted $3 / 0$ silk after return of the colon to the abdomen. The pyogenic membrane was curetted off the chest wall and lung and the chest was closed with two intercostal drains. A small laparotomy was performed to establish a proximal transverse colostomy. Parenteral gentamicin, metronidazole, and cefuroxime were given during and after operation.

The patient's postoperative course was complicated initially by respiratory failure, which required ventilatory support for three days; on the 13 th postoperative day he required drainage of an anterolateral empyema and the thoracotomy wound. This was treated further with daily Eusol-gauze dressings and was resutured after a further eight days. After this he made an uncomplicated recovery. A barium enema on 14 September 1982 showed a sound colonic anastomosis and closure of the colostomy was carried out 44 days after the original procedure.

\section{Discussion}

This case is unique for several reasons. We have been unable to find any other report of tension pneumothorax due to ruptured colon in the chest occurring so long after the original injury to the diaphragm. The only other similar case, described by Das,' occurred 48 hours after direct trauma. Grimes ${ }^{2}$ classifies traumatic rupture of the dia- phragm into three phases: $(a)$ initial or acute, $(b)$ latent, and $(c)$ obstructive.

The case we report here, together with that reported by Das, would suggest the need for inclusion of a fourth phase in the Grimes classification-namely, that of perforation. This would certainly apply to the late intrathoracic perforation described here and is likely to have applied in the case reported by Das, since it is difficult to postulate the migration into the chest of an already perforated segment of colon without concomitant abdominal signs. The differential diagnosis of a tension pneumothorax in this circumstance would include the presence of an air filled but nonperforated viscus such as the colon within the chest, although in such a case the presence of haustrations should assist in making the diagnosis. The stomach may also be present within the thorax and, as Lernau ${ }^{3}$ has pointed out, may present a trap for the unwary; this should be considered in any case presenting as a pneumothorax, particularly if a previous history of trauma has been elicited.

We conclude by suggesting that all sharp injuries to the chest, however superficial they may appear, should be taken seriously. If diaphragmatic rupture is suspected thorough investigation and early operative treatment are mandatory if subsequent and potentially fatal complications are to be prevented.

\section{References}

' Das, PB. Penetrating injury of the diaphragm with herniated colon leaking into the left pleural space. Int Surg 1977;62:463-4.

${ }^{2}$ Grimes OF. Traumatic injuries of the diaphragm. Diaphragmatic hernia. Am J Surg 1974;128:175-81.

${ }^{3}$ Lernau O, Bar-Maor JA, Nissan S. Traumatic diaphragmatic hernia stimulating acute tension pneumothorax. J Trauma 1974;14:880-4. 